The A. L. Mote boen Wients. Nrow Yoot:
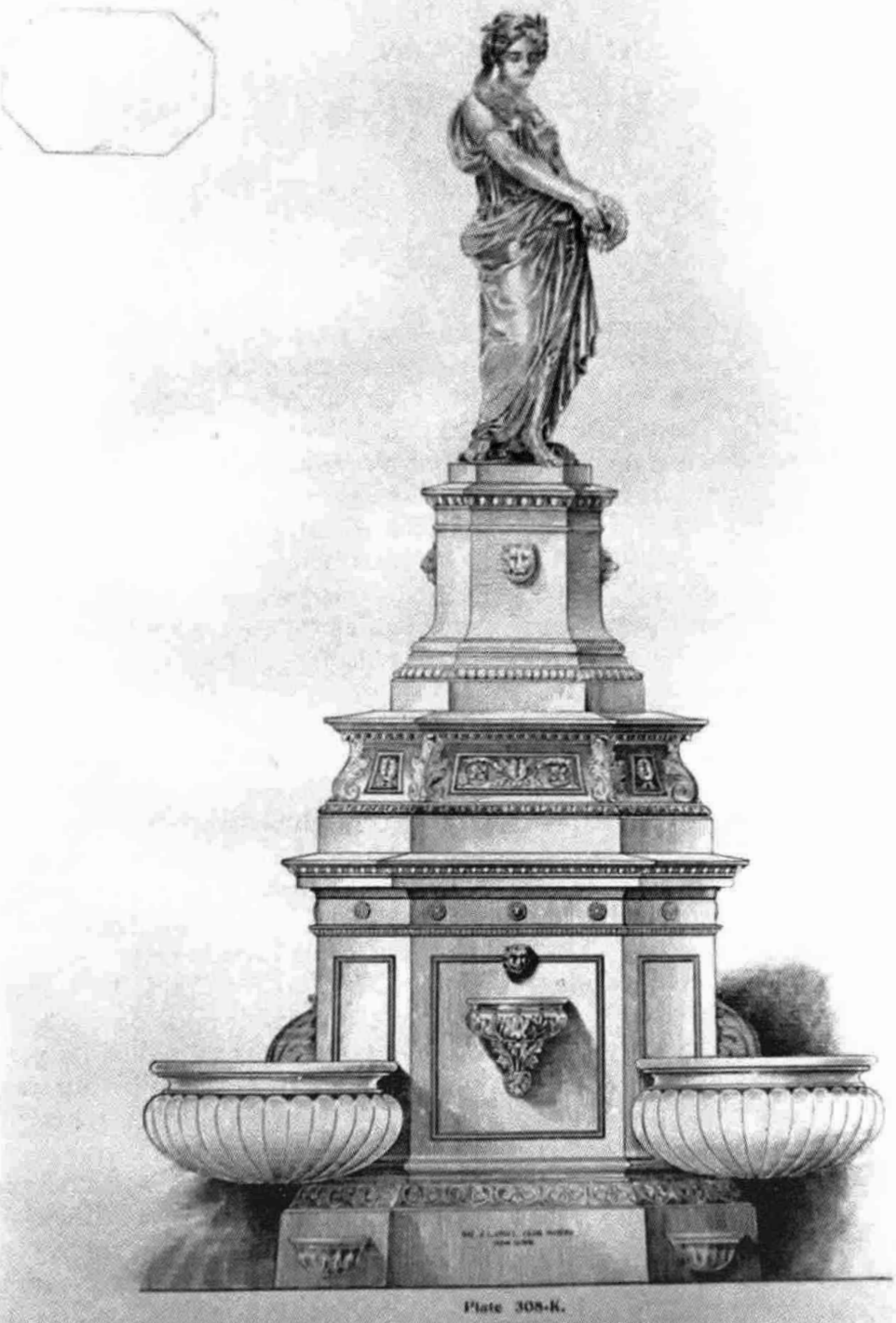

Drinting Fountains with Statue of LA Sounce. 


\title{
El romanticismo y el triunfo de lo impreso
}

\author{
Alain Vaillant
}

A PROPÓSITO DE LA HISTORIA LITERARIA: PRELIMINARES TEÓRICOS

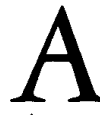
ntes de entrar en el meollo de mi intervención, me parece necesario explicar la perspectiva epistemológica y disciplinaria que me guía. No soy un historiador, sino un especialista en literatura francesa del siglo XIX; más exactamente, me defino como un "historiador de la literatura". Y aun cuando, en Francia, tengo la oportunidad de dialogar frecuentemente con los historiadores, no resulta inútil, con el fin de prevenir algunos malentendidos, explicar en qué sentido mi trayectoria es completamente histórica, por más que trate de objetos particulares cuya especificidad no podría soslayar el historiador de la literatura sin faltar al más elemental rigor científico.

Esquematizando al extremo, podemos decir que, en Francia, han existido durante mucho tiempo dos corrientes principales de historia literaria. La primera corresponde a la concepción más tradicional de la disciplina; su finalidad es hacer la historia, tan exacta y minuciosa como sea posible, de las obras, de los autores y de los movimientos estéticos a los que se supone pertenecen ellos. Los datos históricos tienen ahí su lugar, pero en un segundo plano, como un decorado o, a lo sumo, como un contexto al que es preciso remitirse de vez en cuando. La segunda Corriente, que justamente se fijó como objetivo llenar el vacío que parecía subsistir entre las realidades sociales y los hechos literarios, se desarrolló desde hace algunos decenios, en el momento del gran reflujo de la ola estructuralista sobre los estudios literarios franceses. Esta nueva historia pensó la literatura ya no como una colección de obras singulares, sino en términos de institución o de sistema literario, cuyo examen podía pertenecer a la historia de las representaciones o a la de las sociabilidades, dependiendo de la elección de los literatos para apuntalar sus trabajos con la historia cultural o con la sociología. Pero esta última corriente se hacía a su vez merecedora de un reproche simétrico al que se formuló contra la primera: no ya el de ignorar la historicidad de la literatura, sino por el contrario, el de descuidar la naturaleza artística y formal de las producciones literarias, cuyo análisis histórico pasaba a un segundo plano en provecho de las realidades socioculturales.

Mis trabajos pertenecen a una tercera vía más reciente de la historia literaria, que he contribuido a definir junto con otros. Para empezar, llamaré a esta nueva historia literaria, de la cual han clado testimonio, en el curso de los últimos años, 
varias publicaciones individuales o colectivas, una poética bistórica (poética histórica de las formas y de los géneros). Se trata de estudiar de manera prioritaria la bechura literaria, la evolución de los modos de escritura, las limitaciones formales, los procesos de invención y renovación, y por lo tanto, de colocar en el primer plano las realizaciones textuales en sí, pero integrando las observaciones históricas en apariencia más heterogéneas que quedan excluidas generalmente del campo de la historia literaria. Un ejemplo concreto, tomado del ámbito que justifica mi presencia entre ustedes, a saber, la historia de la prensa, será más elocuente que las largas consideraciones teóricas. Uno de los hechos principales, y reconocido como tal, en la literatura del siglo XIX, es el surgimiento de una práctica generalizada de la brevedad y del texto corto, incluso fragmentario, que culmina, por ejemplo, en la poesía extraordinariamente concentrada de Baudelaire o en la prosa lapidaria de Flaubert. A este respecto, los especialistas tienen la costumbre de concluir que ha nacido una estética de la modernidad, resultante de las transformaciones que la sociedad posrevolucionaria indujo en la percepción del mundo y del lenguaje. Pero no se habían percatado de que este proceso de condensación literaria, siempre asociado con una lógica de ironía, estaba vinculado directamente -lo veremos más ampliamente en mi segunda intervención- con el papel casi hegemónico del periódico en la publicación literaria, hacia las décadas de 1830-1870: el escritor, cuyo texto se pierde en la masa de los otros, queda literariamente constreñido a ser breve y singular, mientras que, hasta entonces, la afirmación de sí, por el contrario, pasaba por la abundancia oratoria.
La modernidad del siglo XIX no puede, por lo tanto, separarse de los nuevos soportes de la publicación que trastocan económica, cultural y estéticamente los mecanismos de la producción literaria.

Como se comprende por medio de este ejemplo: la poética bistórica que yo defiendo debe desembocar en una bistoria de la comunicación literaria, que, a su vez, participa más ampliamente de una historia de las formas de la comunicación social. Éstas abarcaban antaño la conversación, el intercambio epistolar, el manuscrito, la elocuencia pública; hoy en día, habría que añadir a esta lista los medios audiovisuales, el cine, los media electrónicos. En el siglo XIX, la novedad es la nueva función que se atribuye al impreso (sea éste periódico o no), lo cual me permite llegar al cuerpo mismo de mi exposición: "el romanticismo y el triunfo del impreso".

\section{El ROMANTICISMO FRANCÉS \\ Y EL IMPRESO: ESBOZO DE UNA INTERPRETACIÓN HISTÓRICA}

El título que elegí, porque tenía el mérito de la claridad, induciría a error si sólo se enfocara en el aumento masivo de la circulación de los impresos que se produce en el siglo XIX, provocado conjuntamente por los progresos de la alfabetización, la democratización de la lectura y la evolución técnica de la edición. Por una parte, el aumento de los lectores, en lo que se refiere a las obras literarias, es mucho más lento y tardío de lo que se suele imaginar: habrá que esperar el último tercio del siglo XIX para que la literatura -esencialmente la novela- se convierta en un producto de gran consumo; siendo además, 
en la misma época y no antes, cuando aparece la prensa de gran tiraje. Por otra parte y sobre todo, el fenómeno que voy a tratar de describir brevemente ahora tiene alcances de una magnitud mucho más considerable que el solo crecimiento numérico: es cualitativo, y tiene que ver con el funcionamiento mismo de la comunicación literaria.

Más que de un "triunfo del impreso" habría que hablar de un "triunfo de la publicación", entendiendo la palabra "publicación" en su sentido original y preciso. La publicación es el acto de divulgar al "público", es decir, a la esfera pública, entendida como una entidad abstracta, impersonal e indivisible. En el vocabulario del siglo Xxx, el "público" es el equivalente cultural del "pueblo" para los ciudadanos. $\mathrm{Y}$ es precisamente el lugar preponderante que ocupa el impreso en el siglo XIX lo que hace de la comunicación literaria un acto de comunicación pública.

Hasta el siglo XIX, la comunicación literaria es aún un acto privado, efectuado en el interior de un grupo o de una red constituida. Trátese de un manuscrito que se le da a leer a una persona o, por ejemplo, de un poema leído en una tertulia cuyos participantes han sido escogidos, estamos situados en una relación interpersonal, cuyo estudio pertenecería a la sociología de los grupos restringidos y no a un acto de publicación: en esta estructura de redes -de hecho, redes aristocráticas-, la publicación por medio del impreso no interviene más que como un complemento y en la periferia del sistema literario, sea con fines de conservación o de mayor difusión de una obra, y después de que ésta haya circulado manuscrita en el interior de la red, sea para los productos de la cul- tura popular, como son la "literatura azul" o los almanaques. Mi perspectiva, aquí, es por lo tanto complementaria de la de Lise Andries.

Quisiera detenerme por un momento en esta noción de "comunicación literaria pública" por medio del impreso. En primer lugar, hay que apreciar lo que para el escritor puede encerrar de absolutamente excepcional, paradójico y traumático esta comunicación pública de un texto destinado, empero, a la expresión de un pensamiento, de una sensibilidad o de una imaginación singular. No tengo tiempo de desarrollar el examen de esta paradoja más a fondo, pero les pido que lo hagan por su lado, porque ésta es la principal innovación que aporta nuestra cultura moderna del impreso y de ella se derivan todas las demás. En segundo lugar, quisiera subrayar que esta comunicación inmediatamente pública -inmediatamente, es decir, sin la mediación de redes privadas o de grupos restringidos- sólo se hizo posible gracias a la técnica de la impresión: estamos frente a un ejemplo notable de innovación técnicoindustrial que tiene inmensas consecuencias culturales. Finalmente, este nuevo papel de la "publicación" impresa coloca a la literatura en una situación sumamente peculiar en el seno de las prácticas artísticas: con excepción del grabado, las artes plásticas descansan siempre sobre la producción de obras únicas, cuya difusión implica una transacción privada entre un artista y una persona física o moral; la música misma, antes de la invención de las técnicas modernas de grabación, formaba parte de un espectáculo viviente, y por lo tanto, de la reunión de un público congregado en una sala de conciertos. Ésta es, a mi parecer, una de las razones por las que la historia 
del romanticismo literario no puede ser confundida con la de otras expresiones artísticas del romanticismo.

A partir de esta redefinición de la "publicación" literaria, la tesis que quisiera presentar hoy, y que se encuentra en el corazón de todos mis trabajos de historia literaria, es la siguiente: la naturaleza y la evolución del romanticismo francés están estrechamente correlacionadas con la historia de la edición de libros y de periódicos en el siglo XIX. Habrán notado que he hablado de romanticismo francés: no es inútil precisarlo de entrada, puesto que debemos trabajar juntos en un programa de investigación sobre las "transferencias culturales" y el estudio de un fenómeno cultural debe, a mi modo de ver, llevarse a cabo en el marco nacional que le es propio, tomando en consideración todas las especificidades sociohistóricas que recibe de éste. Por supuesto, es posible describir a escala europea una lenta inflexión de la civilización occidental que comienza durante el renacimiento y que culmina, en los siglos XVIII-XIX, con el romanticismo. Éste se define, en una fórmula, como un fenómeno de laicización y de popularización del sentimiento metafísico que se traduce, en el plano filosófico o religioso, por la búsqueda, más allá de las realidades físicas observables, de principios invisibles que permitan explicarlas uniéndolas posteriormente en una síntesis armoniosa entre lo material y lo espiritual; en el plano psicológico, por un acercamiento entre la esfera de la inteligencia racional y de la actividad sensible (sensorial o afectiva) por un lado, y de las realidades subjetivas y objetivas por el otro; en el plano de la Historia y de la acción política, por la voluntad de supeditar la realidad a las exigencias ideales (vo- luntad que se encuentra en el centro del principio revolucionario de 1789 y posteriormente en todas las revoluciones del siglo XIX); en el plano sentimental -que el tríptico anunciante de mi conferencia ha ilustrado muy acertadamente con el motivo de Paul et Virginie (Pablo y Virginia)- por la utopía de la afinidad perfecta entre el cuerpo y el alma de los amantes; en el plano artístico, por la perfecta materialización de un proyecto estético en una forma artística; y, finalmente, en el plano literario, por la elaboración difusa de una verdadera mística de la Voz o del Verbo poético que reúna lo físico y lo metafísico en el acto único de proferir, a imagen de la palabra del Dios cristiano. Todo aquello es cierto, y dibuja efectivamente los contornos de un vasto fenómeno de civilización, cuya huella permanece en el mundo actual. Pero nos quedamos con ello en un nivel de generalidad muy grande. Si queremos avanzar más en la caracterización concreta del romanticismo, es indispensable entrar en el detalle de las historias nacionales, justo lo que me propongo hacer ahora a propósito del romanticismo francés, cuya historia esbozaré en dos tiempos: primero, "la onda de choque de la revolución" y segundo, "el traumatismo de 1830 ". Finalmente, y más allá del ejemplo francés que probablemente no interesa a usťedes directamente, abrigo la esperanza de que mi análisis permitirá elaborar hipótesis o sugerir modelos de interpretación susceptibles de ser generalizados.

\section{LA ONDA DE CHOQUE DE LA REVOLUCIÓN}

De acuerdo con una de las tantas fórmulas lapidarias que abundan en la obra de Víc- 
tor Hugo, el romanticismo es "el resultado de la revolución, sin ser su expresión". Se han señalado múltiples aplicaciones del origen revolucionario del romanticismo. En cambio, se han descuidado generalmente dos características fundamentales que el acontecimiento revolucionario ha aportado indirectamente a la nueva literatura y que, a su vez, inciden en su ideología y en su modo de funcionamiento.

En primer lugar, la revolución inventa un modelo ideal al que el romanticismo no dejará nunca de aspirar después: el del orador revolucionario que, contra toda la tradición del antiguo régimen, vuelve a ubicar la literatura del lado de la elocuencia oratoria y de la comunicación pública. Para Madame de Staël, primera teórica del romanticismo francés, la elocuencia, magnificada por la epopeya revolucionaria, ofrece la síntesis perfecta entre el pensamiento racional y el ámbito de la emoción y del entusiasmo. Si la literatura es, siguiendo la concepción romántica, una alianza perfectamente armoniosa entre lo inteligible y lo sensible, la elocuencia, que en principio supone el colocar la capacidad de convencimiento al servicio de una certeza fundada en la razón, representa el grado supremo de literariedad:

Los progresos de la literatura, es decir, el perfeccionamiento del arte de pensar y de expresarse, son necesarios para establecer y conservar la libertad. [...] Entre los diversos desarrollos del espíritu humano, la literatura filosófica, la elocuencia y el razonamiento son los que considero como la verdadera garantía de la libertad. ${ }^{1}$

${ }^{1}$ Staël, Littérature, 1991, pp. 76-77.
Más allá de esta convicción filosófica, dos razones más precisas explican la presencia del ideal retórico en la ideología revolucionaria.

El discurso del orador es un discurso que se dirige al pueblo, considerado como un principio político, no como una realidad social. La elocuencia revolucionaria no presupone un grupo sociológicamente determinado, como lo era la aristocracia del antiguo régimen, sino que, por el contrario, presume la posibilidad de una universalidad de lo político. Por ello, a todo lo largo del siglo XIX, el orador revolucionario aparece como la encarnación heroica o novelesca del gran escritor. Yo diría incluso que, más allá del romanticismo, esta concepción del acto de escribir como una toma de posición pública inunda toda la gran literatura francesa y permite comprender mejor los vínculos muy estrechos y casi consustanciales que se establecerán en Francia entre literatura y política.

La otra razón que explica el prestigio de la palabra revolucionaria, es que ofrece el modelo, milagrosamente realizado por la Historia, de un discurso dotado de absoluta eficiencia. El orador revolucionario cree que su palabra, si obtiene la adhesión del pueblo (o de sus representantes), es capaz de transformar el mundo, y los acontecimientos parecieron darle la razón, por lo menos provisionalmente. Evidentemente, el sueño de todo escritor es ver su obra producir, sin otra mediación que el tiempo de su escucha o de su enunciación, los efectos más indiscutibles sobre la realidad. No es, pues, una casualidad que el modelo revolucionario haya sido aludido de manera tan recurrente en la historia literaria. La revolución, debido a la confianza que concede estructuralmente, 
por así decirlo, a la palabra y a su capacidad de intervención en el orden de las realidades materiales, se corresponde perfectamente con las modalidades, según las cuales un creador de formas literarias imagina su poder paradójico. Víctor Hugo expresa con la mayor claridad esta oposición entre lo escrito y el discurso público respecto del gran orador revolucionario Mirabeau, con el cual es patente que se siente identificado:

Cuando escribe, Mirabeau es algo menos que Mirabeau [...]. Al leerlo se siente que, lamentablemente, muchas cosas se han quedado en su cabeza [...]. cuando habla, Mirabeau es Mirabeau. Cuando habla, Mirabeau es agua que corre, es ola que produce espuma, es fuego que refulge, es pájaro que vuela, es una cosa que hace su ruido propio, es la naturaleza que cumple su ley. ${ }^{2}$

Quedémonos en esto, por lo que toca al plano de las representaciones y los símbolos. Pero la revolución tuvo sobre la literatura del siglo XIX otro efecto mucho más concreto, inmediato, brutal e irreversible, que pasó totalmente inadvertido aunque se trate, desde mi punto de vista, del fenómeno de mayor relevancia de la historia literaria moderna. Lo acabamos de ver: en el siglo XVIII, aun cuando el desarrollo de la edición hizo progresivamente del libro un competidor del salón aristocrático y del texto impreso un contrapunto de la palabra mundana, las redes privadas de la aristocracia siguieron ocupando el centro de la cultura del antiguo régimen, y el mundo de la edición, su periferia. Por ello la fractura revolucionaria provocó un verdadero sismo en el seno

${ }^{2}$ Ibid, p. 226-227. mismo de la literatura, al destruir de manera casi instantánea estas redes. El intento de restauración que se lleva a cabo bajo el imperio, y sobre todo después de 1815 , no cambiará fundamentalmente esta situación. De hecho, la totalidad del tejido social e interpersonal que aseguraba la comunicación literaria desparece en unas cuantas décadas. Esta circunstancia, totalmente exterior a los movimientos particulares de la historia literaria, tiene como consecuencia una ruptura de brutalidad excepcional en los fenómenos culturales, que explica la absoluta singularidad del romanticismo francés en relación con los demás romanticismos europeos. En efecto, debido a la desaparición de sus estructuras mundanas y privadas, toda la carga del sistema literario recae en el libro y en la comunicación pública. De ahí en adelante, la comunicación literaria se va a resumir en una confrontación heroica entre el escritor y el público, considerado entonces como la emanación del pueblo de los ciudadanos. Jamás se había dotado al acto de publicación literaria de tal poder, de ahí procede la extraordinaria ambición estética y filosófica que anima la literatura romántica francesa confiriéndole el lugar excepcional que ocupa en el seno de la cultura europea, aun cuando los romanticismos inglés y alemán sigan siendo, por su parte, modelos reconocidos y fuentes de inspiración admiradas. Insisto en ello por última vez, porque éste es el centro de mi razonamiento: en un contexto histórico tan particular, el impreso no es solamente un soporte para la difusión de las obras, sino que se integra a la concepción misma que los románticos se forman de la literatura, negando toda legitimidad a su dimensión privada, íntima, dialógica e interpersonal. 


\section{SECUENCIA}
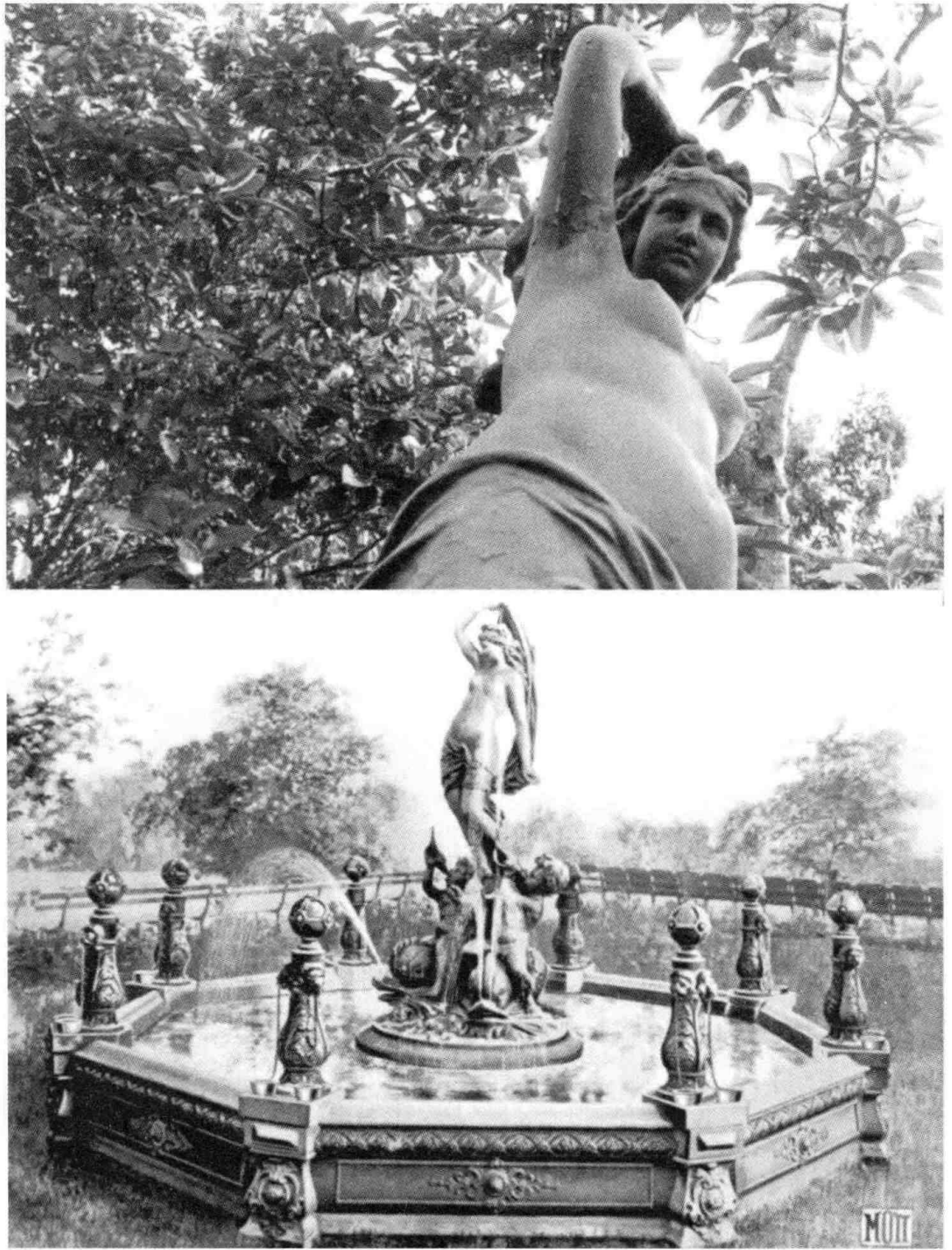
Sin embargo, este diálogo sublime entre el escritor y el público, que la imaginería romántica figura al representarse al "poeta" como un sacerdote o un profeta, no era, evidentemente, más que una ficción, al igual que lo es la del "público", encarnación seductora pero engañosa de un mercado del libro, caracterizado, por el contrario, por su impersonalidad y su anonimato. El escritor creía que de ahí en adelante iba a hablar con todos; y se encuentra solo, reducido a soliloquios. Pensaba que su discurso, liberado del estrecho círculo del mecenazgo, se volvería universal: y no es más que un proveedor de textos, simple engranaje dentro de la industria cultural. Quería ser el gran mediador en el centro del espacio público en vías de constitución: y se vuelve un productor de objetos mediatizados por el mundo complejo del impreso público, periódico o no, que asume de ahí en adelante la función mediadora, organizándola y estandarizándola a través del sistema mediático que entonces se establece. Así llego al segundo tiempo de mi exposición histórica, que llamé el "trauma de 1830".

\section{EL TRAUMA DE 1830}

Ya es tiempo de introducir algo de cronología. El romanticismo triunfante del que acabo de hablar y que corresponde sin duda a la imagen del romanticismo francés que se sigue conservando en el extranjero, duró muy poco tiempo. En términos generales, unos quince años, de 1820 (fecha de publicación de la primera colección de poesías -y también primer best seller romántico-: Las meditaciones, de Lamartine) hasta el comienzo de la década de 1830. Ese romanticismo optimista es el de los poetas de 1820 (Lamartine, Vigny, Hugo) y de los jóvenes artistas, intelectuales, poetas o periodistas que van a participar en la primera de las tres revoluciones del siglo XIX francés: la revolución de julio de 1830 que, a nombre de la libertad de expresión y de publicación, derroca a la vieja dinastía de los Borbones reubicada en el poder después de la caída de Napoleón. Recordemos en efecto que, entre 1814 y 1830, hubo en Francia un intento por volver a la vieja monarquía de derecho divino, durante la cual se sucedieron en el trono los dos hermanos menores de Luis XVI. Este régimen, conocido como "restauración", fue derrocado en 1830 por una oposición que unió a la burguesía liberal y a los republicanos en contra de Carlos X, el hermano más joven de Luis XVI, cuando éste adoptó una línea política particularmente reaccionaria y represiva. Al término del proceso revolucionario, la burguesía y los hombres de negocios fueron quienes se impusieron contra los republicanos y establecieron una monarquía parlamentaria de inspiración liberal, sumamente favorable a los medios económicos ("la monarquía de Julio"). Los escritores, intelectuales y periodistas que habían hecho la revolución con la convicción de que la libertad adquirida aseguraría el triunfo de la nueva literatu$\mathrm{ra}$, van a perder sus ilusiones en el espacio de algunos años, y a adoptar una filosofía desencantada y pesimista, a veces en el límite del nihilismo. Esta reversión fue muy rápida, de una brutalidad verdaderamente excepcional en la historia cultural, que trataré de explicar ahora poniéndola en relación con la nueva cultura del impreso.

Esta cultura descansaba sobre una relación privilegiada e idealizada entre el escritor y el público lector del libro o del 
periódico. Empero, el escritor constató de un modo casi instantáneo el profundo divorcio que existía entre él mismo y este público: de ahí un sentimiento de crisis que, desde entonces, nunca abandonará totalmente la vida literaria francesa, al punto de pasar hoy en día por un simple cliché periodístico, pero que se acentuó entonces en virtud de circunstancias coyunturales.

Por una parte, el público de 1830 estaba tanto menos dispuesto a desempeñar el papel que los románticos querían otorgarle, que un buen número de sus lectores potenciales formaba parte de la nueva burguesía, surgida de las capas más acomodadas del campesinado del antiguo régimen promovidas por la revolución: estos nuevos ricos, a quienes se había mantenido a distancia de la antigua sociedad aristocrática, no tenían ninguna familiaridad con la cultura literaria, y la concepción estrechamente retórica de la enseñanza de la época no podía sino aumentar el sentimiento de incomprensión que experimentaban los escritores. De hecho, el foso entre la burguesía y los medios culturales no se llenará sino hasta fines de siglo, gracias a la acción profunda, eficaz pero muy lenta, de las instituciones educativas y a las reformas pedagógicas: de ahí este "odio al burgués" que es uno de los rasgos más impactantes del romanticismo del siglo XIX y quizás, todavía hoy, de la cultura francesa.

Por otra parte, el mundo del impreso estaba destinado a desempeñar un papel central en el nuevo sistema cultural fundado en la comunicación pública. La edición francesa que había sido tan floreciente económicamente en el siglo XVIII y que tuvo tanta influencia cultural mientras la apuntaló el régimen de "privilegio de li- brería", quedó, sin embargo exangüe, despreciada y desorganizada, después de un cuarto de siglo de revolución y de imperio. Al suprimir el sistema de los privilegios (económicos) sin sustituirlo por ningún otro mecanismo de regulación y protección a la profesión, la revolución, y después el imperio que terminó este trabajo de demolición, sometieron al conjunto de las profesiones del impreso a un control policiaco y a una reglamentación represiva de una temible eficacia. De suerte que la edición francesa, por razones a fin de cuentas totalmente accidentales, se mostró absolutamente incapaz -intelectual, económica o socialmentede conservar el lugar que debe haber sido el suyo en el centro de la vida literaria. No volverá a ser una industria floreciente sino bajo el segundo imperio, y aún habrá que esperar al comienzo del siglo XX para que los grandes editores literarios, siguiendo el modelo de Gaston Gallimard, se conviertan en interlocutores de pleno derecho para el escritor. El libro fracasó entonces para cumplir la función cultural que le correspondía. Por ello, de 1830 a 1870 , el conjunto de la vida cultural francesa se organizará alrededor del periódico. Lo menciono sólo rápidamente porque tendré oportunidad de comentarlo en mi próxima conferencia. A mediados del siglo XIX, el periódico, a causa de su situación casi hegemónica, tiene una importancia desmedida en relación con la que tendrá después, cuando el libro haya retomado su lugar. Este contexto excepcional es lo que vuelve tan necesaria y tan apasionante la historia de la prensa del siglo XIX. Curiosamente, desde hace más de un siglo hemos sido formados de tal manera en el culto al libro y en el desdén a la prensa popular que surge solamente 
a fines del siglo XIX, que aquella relevancia, históricamente capital, pasó más o menos inadvertida hasta hace una década, y es esta aberrante laguna de la historia literaria francesa la que estamos ahora ocupados en llenar.

Pero dejaré aquí, por hoy, el examen detallado del contexto histórico y regresaré a lo esencial, el trauma de 1830 . En su gran mayoría, los medios literarios, intelectuales y artísticos habían sostenido el movimiento de oposición que desembocó en la caída de Carlos X, porque pensaban que con el fin de la restauración desaparecerían los últimos obstáculos que impedían a la palabra literaria alcanzar su público legítimo. Sin duda, los románticos no estaban lejos de pensar que, una vez proclamada la libertad, el público lector y espectador se precipitaría sobre sus colecciones poéticas y sobre sus dramas modernos. No fue así. El público pudo leer, en efecto, y consumir aquello que deseaba; y en tanto público, tenía ganas de informarse y de distraerse.

La nueva libertad -política, pero también económica - trajo consigo, en el espacio de algunos años, una caída brutal de la edición poética, la descalificación cultural del lirismo romántico y un declive del drama de tipo hugoliano, considerado demasiado literario, en beneficio del melodrama o de las grandes máquinas dramáticas al estilo de Dumas, de la novela y, sobre todo, del periódico hecho para instruir o entretener, siendo por supuesto, lo ideal que la novela se incorpore al periódico mediante la invención, en 1836 , de la novela de folletín. Mientras el oficio de escritor periodista se profesionalizaba y constituía, junto con el teatro, la única salida económica del trabajo literario, la literatura -la de los "grandes autores" románticos que leemos aún hoy en día- se sumía en una suerte de depresión larvaria, haciendo ver, desde el fondo de su desesperación, un nihilismo irónico y contestatario. No nos equivoquemos: esta crisis es mucho más que una simple peripecia de la historia cultural: alcanza el funcionamiento y la naturaleza mismos del hecho literario.

Funcionalmente, la crisis cultural que corresponde a la monarquía de Julio suprime el complejo conjunto de mediaciones que se interponía entre el acto singular de la creación y la comunicación pública por medio del impreso. Esta yuxtaposición inmediata de dos lógicas antagónicas -digamos, si se quiere, la lógica del uno y la de lo múltiple- es absolutamente nueva en la historia literaria. Los escritores románticos se dan cuenta de que la ausencia de estructura mediadora los coloca en una situación concreta insostenible si no se limitan a responder a la demanda del mercado. En un plano personal e íntimo, alimentan un sentimiento de malestar, de soledad o de incomprensión del que darán testimonio sus escritos íntimos o, por la vía de diversos filtros genéricos, las obras literarias mismas. De suerte que toda la historia posterior de las instituciones literarias puede resumirse en los diferentes esfuerzos encaminados a reconstituir sistemas oficiosos e incluso clandestinos de mediación, con el fin de recrear, al margen de la comunicación pública, un tejido de relaciones interpersonales, trátese de la bohemia romántica, de las sociedades de autores, de las vanguardias, de los rituales de la camaradería literaria, de la reaparición de formas disfrazadas de mecenazgo público (especialmente por medio de las instituciones escolares o culturales), o del papel cen- 
tral que tuvieron, en el siglo $\mathrm{xx}$, los grandes editores literarios que instituyeron a su vez una suerte de mecenazgo interesado.

De manera más fundamental aún, la naturaleza misma de la literatura es la que se trastoca. Según se va estableciendo un sistema complejo -público, industrial y normalizado- de producción y de circulación de lo impreso, el hecho literario deja de definirse como un acto de mediación, un discurso que saca su valor de su función en el seno de los intercambios intersubjetivos al mismo tiempo que de la toma de conciencia estética que se desprende de ellos para volverse un objeto, a su vez, mediatizado, un texto apoyado por los mecanismos de la cultura mediática y sometido, por otra parte, a las reglas económicas del capitalismo moderno. En oposición a la "literatura-discurso", he sugerido nombrar "literatura-texto" a este nuevo régimen de literariedad, que se vuelve desde entonces el nuestro. Este proceso de textualización de la literatura tiene consecuencias incalculables en el arte de la escritura y en la poética de los géneros. Sólo señalaré aquí, para no entrar en debates demasiado literarios, un fenómeno, el más espectacular: la novela -libro de ficción excluido desde sus orígenes, y con toda razón, de la tradición oratoriase convierte en el género dominante de la literatura y sustituye la poesía.

Está claro que, aun cuando exista un sentimiento real de crisis, que es parte integrante del imaginario romántico, también nace entonces una nueva cultura que va a expandirse en el universo del periódico; ya tendré oportunidad de volver a ello. Por hoy, sólo quisiera añadir dos palabras más de conclusión.

Una consigna metodológica: el siglo XIX conoce una ruptura cultural cuya novedad no debe, a mi parecer, minimizarse.
Pero para apreciar esta novedad, es necesario realizar un trabajo teórico y conceptual cuyos contornos he tratado de esbozar vagamente.

Una observación más histórica. En el fondo, la singularidad del romanticismo francés puede resumirse en pocas palabras. En unos cuantos años -entre 1820 y 1840: el tiempo de una generación- los medios culturales, sin estar preparados para ello, sufren el vuelco del mundo de las bellas letras ttadicionales a nuestro sistema (literario) actual, fundado en el mercado público, el régimen mediático y las industrias culturales. La brutalidad de esta ruptura, que obliga a los escritores a imaginarlo todo, explica la excepcional riqueza cultural de esta época y la inventiva incomparable de sus creadores: de hecho, lo que se produce es un estado transitorio de anomia en el cual todo parece posible, lo mejor y lo peor. Para nosotros, los investigadores, este periodo constituye una especie de laboratorio histórico que permite observar, de manera mucho más clara, unos mecanismos culturales que muy pronto van a hacerse más complejos y confusos: por eso creo, con mucha firmeza, que la historia del romanticismo francés ofrece la posibilidad de concebir un modelo de análisis histórico que deberá confrontarse con otras épocas (por ejemplo con el mundo de hoy) o con otras áreas geográficas. Y estoy seguro de que nuestra cooperación en torno a la prensa mexicana del siglo XIX nos dará la oportunidad de llevar a cabo aquel diálogo, lo cual me alegra.

\section{BIBLIOGRAFÍA}

-Staël, Germaine de, De la littérature considérée dans ses rapports avec les institutions sociales, GF, París, 1991. 